\title{
On the stretch factor of the Theta-4 graph
}

\author{
Luis Barba*† Prosenjit Bose* Jean-Lou De Carufel* \\ André van Renssen* Sander Verdonschot*
}

\begin{abstract}
In this paper we show that the $\theta$-graph with 4 cones has constant stretch factor, i.e., there is a path between any pair of vertices in this graph whose length is at most a constant times the Euclidean distance between that pair of vertices. This is the last $\theta$-graph for which it was not known whether its stretch factor was bounded.
\end{abstract}

\section{Introduction}

A $t$-spanner of a weighted graph $G$ is a connected sub-graph $H$ with the property that for all pairs of vertices $u$ and $v$, the weight of the shortest path between $u$ and $v$ in $H$ is at most $t$ times the weight of the shortest path between $u$ and $v$ in $G$, for some fixed constant $t \geq 1$. The smallest constant $t$ for which $H$ is a $t$-spanner of $G$ is referred to as the stretch factor or spanning ratio of the graph. The graph $G$ is referred to as the underlying graph. In our setting, the underlying graph is the complete graph on a set of $n$ points in the plane and the weight of an edge is the Euclidean distance between its endpoints. A spanner of such a graph is called a geometric spanner. For a comprehensive overview of geometric spanners, see the book by Narasimhan and Smid [8].

In this paper, we focus on $\theta$-graphs. Introduced independently by Clarkson [5] and Keil [7, the $\theta_{m^{-}}$ graph is constructed as follows. Given a set $P$ of points in the plane, we consider each point $p \in P$ and partition the plane into $m$ cones (regions in the plane between two rays originating from the same point) with apex $p$, each defined by two rays at consecutive multiples of $\theta=2 \pi / m$ radians from the negative $y$-axis. We label the cones $C_{0}(p)$ through $C_{m-1}(p)$, in counter-clockwise order around $p$, starting from the negative y-axis; see Fig. 1. In each cone $C_{i}(p)$, we add an edge between $p$ and $p_{i}$, the point in $C_{i}(p)$ nearest to $p$. However, instead of using the Euclidean distance, we measure distance in $C_{i}(p)$ by projecting

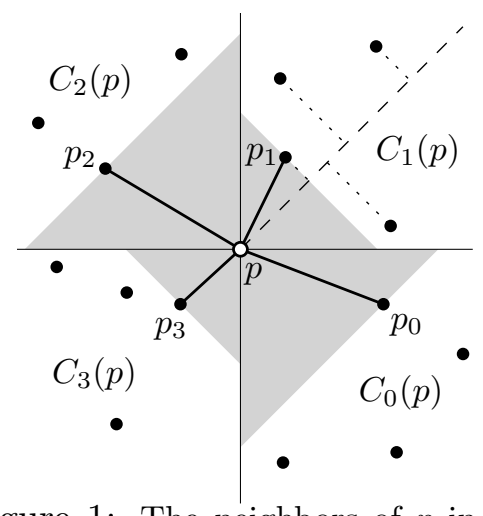

Figure 1: The neighbors of $p$ in the $\theta_{4}$-graph of $P$. Each edge supports an empty isosceles triangle. each vertex onto the angle bisector of this cone. Formally, $p_{i}$ is the point in $C_{i}(p)$ such that for every other point $w \in C_{i}(p)$, the projection of $p_{i}$ onto the angle bisector of $C_{i}(p)$ lies closer to $p$ than that of $w$. For simplicity, we assume that no two points of $P$ lie on a line parallel to either the boundary or the angle bisector of a cone.

\footnotetext{
${ }^{*}$ Carleton University, Ottawa, Canada

${ }^{\dagger}$ Université Libre de Bruxelles, Brussels, Belgium
} 
Ruppert and Seidel [9] showed that $\theta_{m}$-graphs are geometric spanners for $m \geq 7$, and their stretch factor approaches 1 as $m$ goes to infinity. Their proof crucially relies on the fact that, given two vertices $p$ and $q$ such that $q \in C_{i}(p)$, the distance between $p_{i}$ and $q$ is always less than the distance between $p$ and $q$. This property does not hold for $m \leq 6$ and indeed, the path obtained by starting at $p$ and repeatedly following the edge in the cone that contains $q$, is not necessarily a spanning path. The main motivation for using spanners is usually to reduce the number of edges in the graph without increasing the length of shortest paths too much. Thus, $\theta$-graphs with fewer cones are more interesting in practice, as they have fewer edges. This raises the following question: "What is the smallest $m$ for which the $\theta_{m}$-graph is a geometric spanner?" Bonichon et al. [1 showed that the $\theta_{6}$-graph is also a geometric spanner. Recently, Bose et al. [4] proved the same for the $\theta_{5}$-graph. Coming from the other side, El Molla [6] showed that there is no constant $t$ for which the $\theta_{2^{-}}$and $\theta_{3}$-graphs are geometric spanners. This leaves the $\theta_{4}$-graph as the only open question. Moreover, its resemblance to graphs like the $\mathrm{YaO}_{4}$-graph [3] and the $L_{\infty}$-Delaunay triangulation [2], both of which are spanners, make this question more tantalizing. In this paper we establish an upper bound of approximately 237 on the stretch factor of the $\theta_{4}$-graph, thereby showing that it is a geometric spanner. In Section 5 , we present a lower bound of 7 that we believe is closer to the true stretch factor of the $\theta_{4}$-graph.

\section{Existence of a spanning path}

Let $P$ be a set of points in the plane. In this section, we prove that the $\theta_{4}$-graph of $P$ is a spanner. We do this by showing that the $\theta_{4}$-graph approximates the $L_{\infty}$-Delaunay triangulation. The $L_{\infty}$-Delaunay triangulation of $P$ is a geometric graph with vertex set $P$, and an edge between two points of $P$ whenever there exists an empty axis-aligned square having these two points on its boundary.

Bonichon et al. [2] showed that the $L_{\infty}$-Delaunay triangulation has a stretch factor of $c^{*}=\sqrt{4+2 \sqrt{2}}$, i.e., there is a path between any two vertices whose length is at most $c^{*}$ times their Euclidean distance. We approximate this path in the $L_{\infty}$-Delaunay triangulation by showing the existence of a spanning path in the $\theta_{4}$-graph of $P$ joining the endpoints of every edge in the $L_{\infty}$-Delaunay triangulation. The main ingredient to obtain this approximation is Lemma 1 whose proof is deferred to Section 4 . Before we can state this lemma, we need a few more definitions. Given two points $s$ and $t$, their $L_{1}$ distance $d_{L_{1}}(s, t)$ is the sum of the absolute differences of their $x$ - and $y$-coordinates.

Let $S_{t}(s)$ be the smallest axis-aligned square centered on $t$ that contains $s$. Let $\ell_{t}^{-}$and $\ell_{t}^{+}$be the lines with slope -1 and +1 passing through $t$, respectively.

Throughout this paper, we repeatedly use $t$ to denote a target point of $P$ that we want to reach via a path in the $\theta_{4}$-graph. Therefore, we typically omit the reference to $t$ and write $\ell^{-}, \ell^{+}$and $S(s)$ when referring to $\ell_{t}^{-}, \ell_{t}^{+}$and $S_{t}(s)$, respectively.

We say that an object is empty if its interior contains no point of $P$. An $s$-t-path is a path with endpoints $s$ and $t$.

Lemma 1. Let $s$ and $t$ be two points of $P$ such that $t$ lies in $C_{0}(s)$. If the top-right quadrant of $S(s)$ is empty and $C_{1}(s)$ contains no point of $P$ below $\ell^{-}$, then there is an $s$-t-path in the $\theta_{4}$-graph of $P$ of length at most $18 \cdot d_{L_{1}}(s, t)$.

Given a path $\varphi$, let $|\varphi|$ denote the sum of the lengths of the edges in $\varphi$. Using Lemma 1 . we obtain the following. 
a)

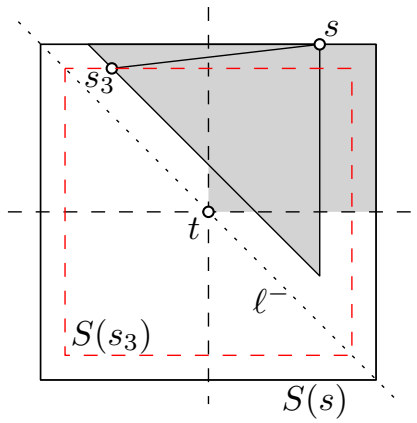

b)

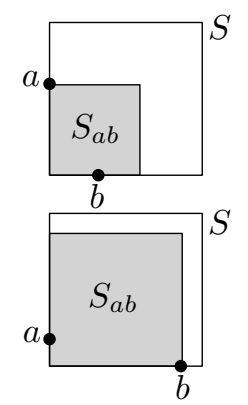

Figure 2: a) Configuration used in the proof of Lemma 2, grey areas represent empty regions. $b$ ) If $a$ and $b$ lie on consecutive sides of a square $S$, there is a square $S_{a b}$ such that $a b \subset S_{a b} \subseteq S$ and either $a$ or $b$ lies on a corner of $S_{a b}$.

Lemma 2. Let $s$ and $t$ be two points of $P$. If the smallest axis-aligned square enclosing $s$ and $t$, that has $t$ as a corner, is empty, then there is an $s$-t-path in the $\theta_{4}$-graph of $P$ of length at most $(\sqrt{2}+36) \cdot|s t|$.

Proof. Assume without loss of generality that $s$ lies in $C_{1}(t)$. Then, the top-right quadrant of $S(s)$ is empty as it coincides with the smallest axis-aligned square enclosing $s$ and $t$ that has $t$ as a corner; see Fig. 2(a). Recall that $s_{3}$ is the neighbor of $s$ in the $\theta_{4}$-graph inside the cone $C_{3}(s)$. Assume that $s_{3} \neq t$ as otherwise the result follows trivially. Consequently, $s_{3}$ must lie either in $C_{0}(t)$ or in $C_{2}(t)$. Assume without loss of generality that $s_{3}$ lies in the top-left quadrant of $S(s)$. As $s_{3}$ lies in the interior of $S(s), S\left(s_{3}\right) \subset S(s)$ and hence, the top-right quadrant of $S\left(s_{3}\right)$ is empty. Moreover, $s_{3}$ lies above $\ell^{-}$and hence $C_{1}\left(s_{3}\right)$ contains no point of $P$ below $\ell^{-}$. Therefore, by Lemma 1 there is an $s_{3}$-t-path $\varphi$ of length at most $18 \cdot d_{L_{1}}\left(s_{3}, t\right)$. Since $s_{3}$ lies inside $S(s),\left|s_{3} t\right| \leq \sqrt{2} \cdot|s t|$ and hence $|\varphi| \leq 18 \cdot d_{L_{1}}\left(s_{3}, t\right) \leq 18 \sqrt{2} \cdot\left|s_{3} t\right| \leq 18 \sqrt{2} \sqrt{2} \cdot|s t|=36 \cdot|s t|$. Moreover, the length of edge $s s_{3}$ is at most $d_{L_{1}}(s, t) \leq \sqrt{2} \cdot|s t|$ since $s_{3}$ must lie above $\ell^{-}$. Thus, $s s_{3} \cup \varphi$ is an $s$-t-path of length $\left|s s_{3}\right|+|\varphi| \leq(\sqrt{2}+36) \cdot|s t|$.

The following observation is depicted in Fig. 2(b).

Observation 3. Let $S$ be an axis-aligned square. If two points a and $b$ lie on consecutive sides along the boundary of $S$, then there is a square $S_{a b}$ containing the segment ab such that $S_{a b} \subseteq S$ and either a or $b$ lies on a corner of $S_{a b}$.

Lemma 4. Let $a b$ be an edge of the $L_{\infty}$-Delaunay triangulation of $P$. There is an $a-b$-path $\varphi_{a b}$ in the $\theta_{4}$-graph of $P$ such that $\left|\varphi_{a b}\right| \leq(1+\sqrt{2}) \cdot(\sqrt{2}+36) \cdot|a b|$.

Proof. Let $T=(a, b, c)$ be a triangle in the $L_{\infty}$-Delaunay triangulation of $P$. By definition of this triangulation, there is an empty square $S$ such that every vertex of $T$ lies on the boundary of $S$. By the general position assumption, $a, b$ and $c$ must lie on different sides of $S$. If $a$ and $b$ lie on consecutive sides of the boundary of $S$, then by Observation 3 and Lemma 2 there is a path $\varphi_{a b}$ contained in the $\theta_{4}$-graph of $P$ such that $\left|\varphi_{a b}\right| \leq(\sqrt{2}+36) \cdot|a b|$.

If $a$ and $b$ lie on opposite sides of $S$, then both $a c$ and $c b$ have their endpoints on consecutive sides along the boundary of $S$. Let $S_{a c}$ be the square contained in $S$ existing as a consequence of Observation 3 when applied on the edge $a c$. Thus, either $a$ or $c$ lies on a corner of $S_{a c}$. Furthermore, as $S_{a c}$ is contained in $S$, it is also empty. Consequently, by Lemma 2, there is a $a-c$-path $\varphi_{a c}$ such that $\left|\varphi_{a c}\right| \leq(\sqrt{2}+36) \cdot|a c|$. Analogously, there is a path $\varphi_{c b}$ such that $\left|\varphi_{c b}\right| \leq(\sqrt{2}+36) \cdot|c b|$. Using elementary geometry, it can be shown 
that since $a$ and $b$ lie on opposite sides of $S,|a c|+|c b| \leq(1+\sqrt{2}) \cdot|a b|$. Therefore, the path $\varphi_{a b}=\varphi_{a c} \cup \varphi_{c b}$ is an $a$-b-path such that $\left|\varphi_{a b}\right| \leq(1+\sqrt{2}) \cdot(\sqrt{2}+36) \cdot|a b|$.

Theorem 5. The $\theta_{4}$-graph of $P$ is a spanner whose stretch factor is at most

$$
(1+\sqrt{2}) \cdot(\sqrt{2}+36) \cdot \sqrt{4+2 \sqrt{2}} \approx 237
$$

Proof. Let $\nu$ be the shortest path joining $s$ with $t$ in the $L_{\infty}$-Delaunay triangulation of $P$. Bonichon et al. 2] proved that the length of $\nu$ is at most $\sqrt{4+2 \sqrt{2}} \cdot|s t|$. By replacing every edge in $\nu$ with the path in the $\theta_{4}$-graph of $P$ that exists by Lemma 4 , we obtain an $s$-t-path of length at most

$$
(1+\sqrt{2}) \cdot(\sqrt{2}+36) \cdot|\nu| \leq(1+\sqrt{2}) \cdot(\sqrt{2}+36) \cdot \sqrt{4+2 \sqrt{2}} \cdot|s t|
$$

\section{Light paths}

We introduce some tools that will help us proving Lemma 1 in Section 4 .

Given a point $p$ of $P$, we call edge $p p_{i}$ an $i$-edge. Let $\varphi$ be a path that follows only 0and 1-edges. A 0 -edge $p p_{0}$ of $\varphi$ is light if no edge of $\varphi$ crosses the horizontal ray shooting to the right from $p$. We say that $\varphi$ is a light path if all its 0-edges are light. In this section we show how to bound the length of a light path with respect to the Euclidean distance between its endpoints.

Notice that every $i$-edge is associated with an empty isosceles right triangle. For a point $p$, the empty triangle generated by its $i$-edge is denoted by $\Delta_{i}(p)$.

Lemma 6. Given a light path $\varphi$, every pair of 0 -edges of $\varphi$ has disjoint orthogonal projection on the line defined by the equation $y=-x$.

Proof. Let $s$ and $t$ be the endpoints of $\varphi$. Let $p p_{0}$ be any 0 -edge of $\varphi$ and let $\nu_{p_{0}}$ be the diagonal line extending the hypotenuse of $\Delta_{0}(p)$, i.e., $\nu_{p_{0}}$ is a line with slope +1 passing through $p_{0}$. Let $\gamma$ be the path contained in $\varphi$ that joins $p_{0}$ with $t$. We claim that every point in $\gamma$ lies below $\nu_{p_{0}}$. If this claim is true, the diagonal lines constructed from the empty triangles of every 0 -edge in $\varphi$ split the plane into disjoint slabs, each containing a different 0 -edge of $\varphi$. Thus, their projection on the line defined by the equation $y=-x$ must be disjoint.

To prove that every point in $\gamma$ lies below $\nu_{p_{0}}$, notice that every point in $\gamma$ must lie to the right of $p$ since $\varphi$ is $x$-monotone, and below $p$ since $p p_{0}$ is light, i.e., $\gamma$ is contained in $C_{0}(p)$. Since $\Delta_{0}(p)$ is empty, no point of $\gamma$ lies above $\nu_{p_{0}}$ and inside $C_{0}(p)$ yielding our claim.

Given a point $w$ of $P$, we say that a point $p$ of $P$ is $w$-protected if $C_{1}(p)$ contains no point of $P$ below or on $\ell_{w}^{-}$, recall that $\ell_{w}^{-}$is the line with slope -1 passing through $w$. In other words, a point $p$ is $w$-protected if either $C_{1}(p)$ is empty or $p_{1}$ lies above $\ell_{w}^{-}$. Moreover, every point lying above $\ell_{w}^{-}$is $w$-protected and no point in $C_{3}(w)$ is $w$-protected.

Given two point $s$ and $t$ such that $s$ lies to the left of $t$, we aim to construct a path joining $s$ with $t$ in the $\theta_{4}$-graph of $P$. The role of $t$-protected points will be central in this construction. However, as a first step, we relax our goal and prove instead the existence of a light path $\sigma_{s \rightarrow t}$ going from $s$ towards $t$ that does not necessarily end at $t$.

To construct $\sigma_{s \rightarrow t}$, start at a point $z=s$ and repeat the following steps until reaching either $t$ or a $t$-protected point $w$ lying to the right of $t$.

- If $z$ is not $t$-protected, then follow its 1-edge, i.e., let $z=z_{1}$. 
- If $z$ is $t$-protected, then follow its 0 -edge, i.e., let $z=z_{0}$.

The pseudocode of this algorithm can be found in Algorithm 1 .

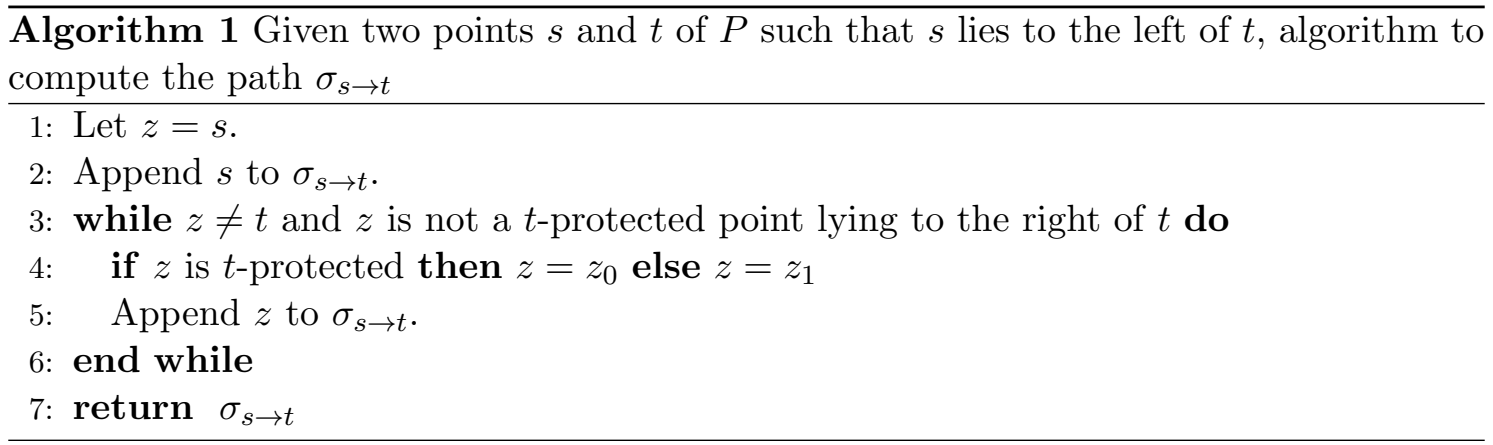

Lemma 7. Let $s$ and $t$ be two points of $P$ such that $s$ lies to the left of $t$. Algorithm 1 produces a light $x$-monotone path $\sigma_{s \rightarrow t}$ joining s with a t-protected point $w$ such that either $w=t$ or $w$ lies to the right of $t$. Moreover, every edge on $\sigma_{s \rightarrow t}$ is contained in $S(s)$.

Proof. By construction, Algorithm 1 finishes only when reaching either $t$ or a $t$-protected point lying to the right of $t$. Since every edge of $\sigma_{s \rightarrow t}$ is either a 0 -edge or a 1 edge traversed from left to right, $\sigma_{s \rightarrow t}$ is $x$-monotone.

The left endpoint of every 0-edge in $\sigma_{s \rightarrow t}$ lies in $C_{2}(t)$ as it most be $t$-protected and no $t$-protected point lies in $C_{3}(t)$. Thus, if $v v_{0}$ is a 0-edge, then $v$ lies in $C_{2}(t)$ and hence, $v_{0}$ lies inside $S(s)$ and above $\ell^{+}$. Otherwise $t$ would lie inside $\Delta_{0}(v)$. Therefore, every 0-edge in $\sigma_{s \rightarrow t}$ is contained in $S(s)$.

Every 1-edge in $\sigma_{s \rightarrow t}$ has its two endpoints lying below $\ell^{-}$; otherwise, we followed the 1-edge of a $t$ -

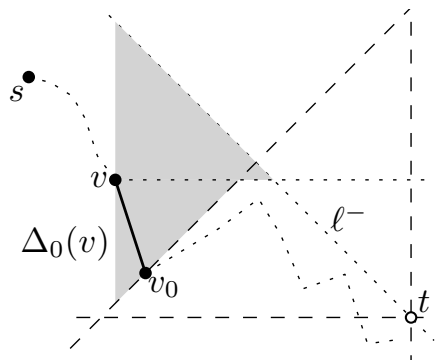

Figure 3: If $v$ is a $t$-protected point, then edge $v v_{0}$ is light in any path $\sigma_{s \rightarrow t}$ that contains it. protected point which is not allowed by Step 4 of Algorithm 1. Thus, every 1-edge in $\sigma_{s \rightarrow t}$ lies below $\ell^{-}$and to the right of $s$. As 1-edges are traversed from bottom to top and the 0-edges of $\sigma_{s \rightarrow t}$ are enclosed by $S(s)$, every 1-edge in $\sigma_{s \rightarrow t}$ is contained in $S(s)$.

Let $v v_{0}$ be any 0-edge of $\sigma_{s \rightarrow t}$. Since we followed the 0-edge of $v$, we know that $v$ is $t$-protected and hence no point of $P$ lies in $C_{1}(v)$ and below $\ell^{-}$. As every 1-edge has its two endpoints lying below $\ell^{-}$and $\sigma_{s \rightarrow t}$ is $x$-monotone, no 1-edge in $\sigma_{s \rightarrow t}$ can have an endpoint in $C_{1}(v)$. In addition, every 0-edge of $\sigma_{s \rightarrow t}$ joins its left endpoint with a point below it. Thus, no 0-edge of $\sigma_{s \rightarrow t}$ can cross the ray shooting to the right from $v$. Consequently, $v v_{0}$ is light and hence $\sigma_{s \rightarrow t}$ is a light path; see Fig 3 .

Given two points $p$ and $q$, let $|p q|_{x}$ and $|p q|_{y}$ be the absolute differences between their $x$ - and $y$-coordinates, respectively, i.e., $d_{L_{1}}(p, q)=|p q|_{x}+|p q|_{y}$.

Lemma 8. Let $s$ and $t$ be two points of $P$ such that $s$ lies to the left of $t$. If $s$ is t-protected, then $\left|\sigma_{s \rightarrow t}\right| \leq 3 \cdot d_{L_{1}}(s, t)$.

Proof. To bound the length of $\sigma_{s \rightarrow t}$, we bound the length of its 0-edges and the length of its 1-edges separately. Let $Z$ be the set of all 0-edges in $\sigma_{s \rightarrow t}$ and consider their orthogonal projection on $\ell^{-}$. By Lemma 6, all these projections are disjoint. Moreover, the length of every 0 -edge in $Z$ is at most $\sqrt{2}$ times the length of its projection. Let $s_{\perp}$ be the 

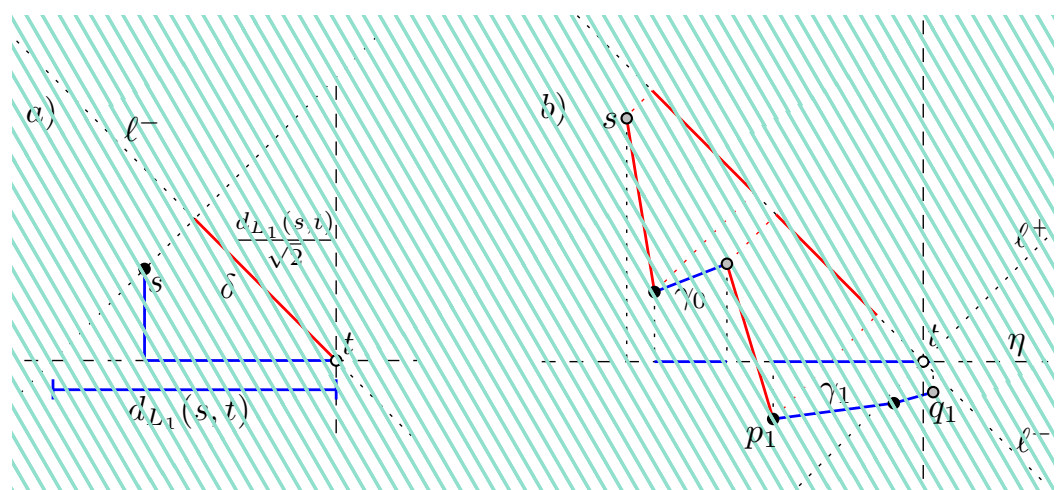

Figure 4: a) The segment $\delta$ having length $d_{L_{1}}(s, t) / \sqrt{2}$. b) The 0-edges of $\sigma_{s \rightarrow t}$ have disjoint projections on $\ell^{-}$and the 1-edges have disjoint projections on the horizontal line passing through $t$. The slope between the endpoints of the maximal paths $\gamma_{0}$ and $\gamma_{1}$ is less than 1 .

orthogonal projection of $s$ on $\ell^{-}$and let $\delta$ be the segment joining $s_{\perp}$ with $t$. Since $s$ is $t$-protected and $\sigma_{s \rightarrow t}$ is $x$-monotone, the orthogonal projection of every 0-edge of $Z$ on $\ell^{-}$ is contained in $\delta$ and hence $\sum_{e \in Z}|e| \leq \sqrt{2} \cdot|\delta|$. Since $|\delta|=d_{L_{1}}(s, t) / \sqrt{2}$ as depicted in Fig. 4(a), we conclude that $\sum_{e \in Z}|e| \leq d_{L_{1}}(s, t)$.

Let $O$ be the set of all 1-edges in $\sigma_{s \rightarrow t}$ and let $\eta$ be the horizontal line passing through $t$. Since $\sigma_{s \rightarrow t}$ is $x$-monotone, the orthogonal projections of all edges in $O$ on $\eta$ are disjoint. Let $\gamma_{0}, \ldots, \gamma_{k}$ be the connected components induced by $O$, i.e., the set of maximal connected paths that can be formed by the 1-edges in $O$; see Fig. 4(b). We claim that the slope of the line joining the two endpoints $p^{i}, q^{i}$ of every $\gamma_{i}$ is smaller than 1 . If this claim is true, the length of every $\gamma_{i}$ is bounded by $\left|p^{i} q^{i}\right|_{x}+\left|p^{i} q^{i}\right|_{y} \leq 2 \cdot\left|p^{i} q^{i}\right|_{x}$ as each $\gamma_{i}$ is $x$ - and $y$-monotone.

To prove that the slope between $p^{i}$ and $q^{i}$ is smaller than 1 , let $v v_{0}$ be the 0 -edge of $\sigma_{s \rightarrow t}$ such that $v_{0}=p^{i}$. Since $v v_{0}$ is in $\sigma_{s \rightarrow t}, v$ is $t$-protected by Step 4 of Algorithm 1 and hence, as $\Delta_{0}(v)$ is empty, $q^{i}$ must lie below the line with slope +1 passing through $p^{i}$ yielding our claim.

Let $\omega$ be the segment obtained by shooting a ray from $t$ to the left until hitting the boundary of $S(s)$. We bound the length of all edges in $O$ using the length of $\omega$. Notice that the orthogonal projection of every $\gamma_{i}$ on $\eta$ is contained in $\omega$, except maybe for $\gamma_{k}$ whose right endpoint $q^{k}$ could lie below and to the right of $t$. Two cases arise: If the projection of $\gamma_{k}$ on $\eta$ is contained in $\omega$, then $\sum_{i=0}^{k}\left|\gamma_{i}\right| \leq \sum_{i=0}^{k} 2 \cdot\left|p^{i} q^{i}\right|_{x} \leq 2 \cdot|\omega|$. Otherwise, since $q_{k}$ is $t$-protected, $q_{k}$ lies below $\ell^{-}$and hence $d_{L_{1}}\left(p^{k}, q^{k}\right) \leq d_{L_{1}}\left(p^{k}, t\right)$. Moreover, $p^{k}$ must lie above $\ell^{+}$as $p^{k}$ is reached by a 0 -edge coming from above $\eta$, i.e., $\left|p^{k} t\right|_{y}<\left|p^{k} t\right|_{x}$. Therefore,

$$
\left|\gamma_{k}\right| \leq d_{L_{1}}\left(p^{k}, q^{k}\right) \leq d_{L_{1}}\left(p^{k}, t\right)=\left|p^{k} t\right|_{x}+\left|p^{k} t\right|_{y} \leq 2 \cdot\left|p^{k} t\right|_{x}
$$

Consequently, $\sum_{i=0}^{k}\left|\gamma_{i}\right| \leq 2 \cdot\left|p^{k} t\right|_{x}+\sum_{i=0}^{k-1} 2 \cdot\left|p^{i} q^{i}\right|_{x} \leq 2 \cdot|\omega|$. Since $|\omega| \leq d_{L_{1}}(s, t)$, we get that $\sum_{e \in O}|e|=\sum_{i=0}^{k}\left|\gamma_{i}\right| \leq 2 \cdot d_{L_{1}}(s, t)$. Thus, $\sigma_{s \rightarrow t}$ is a light path of length at most $\sum_{e \in O}|e|+\sum_{e \in Z}|e| \leq 3 \cdot d_{L_{1}}(s, t)$.

By the construction of the light path in Algorithm 1, we observe the following.

Lemma 9. Let $s$ and $t$ be two points of $P$ such that $s$ lies to the left of $t$. If the right endpoint $w$ of $\sigma_{s \rightarrow t}$ is not equal to $t$, then $w$ lies either above $\ell^{+}$if $w \in C_{1}(t)$, or below $\ell^{-}$ if $w \in C_{0}(t)$. 
Proof. If $w$ lies in $C_{1}(t)$, then by Step 4 of Algorithm 1, $w$ was reached by a 0-edge $p w$ such that $p$ is a $t$-protected point lying above and to the left of $t$. As $\Delta_{0}(p)$ is empty, $t$ lies below the hypotenuse of $\Delta_{0}(p)$ and hence $w$ lies above $\ell^{+}$.

Assume that $w$ lies in $C_{0}(t)$. Notice that $w$ is the only $t$-protected point of $\sigma_{s \rightarrow t}$ that lies to the right of $t$; otherwise, Algorithm 1 finishes before reaching $w$. By Step 4 of Algorithm 1, every 0 -edge of $\sigma_{s \rightarrow t}$ needs to have a $t$-protected left endpoint. Moreover, every $t$-protected point of $\sigma_{s \rightarrow t}$, other that $w$, lies above and to the left of $t$. Therefore, $w$ is not reached by a 0-edge of $\sigma_{s \rightarrow t}$, i.e., $w$ must be the right endpoint of a 1-edge $p w$ of $\sigma_{s \rightarrow t}$. Notice that $w$ cannot lie above $\ell^{-}$since otherwise $p$ is $t$-protected and hence Algorithm 1 finishes before reaching $w$ yielding a contradiction. Thus, $w$ lies below $\ell^{-}$.

\section{One empty quadrant}

In this section, we provide the proof of Lemma 1. Before stepping into the proof, we need one last definition. Given a point $p$ of $P$, the $\max _{1}$-path of $p$ is the longest path having $p$ as an endpoint that consists only of 1-edges and contains the edge $p p_{1}$. We restate Lemma 1 using the notions of $t$-protected and $s$-t-path.

Lemma 1. Let $s$ and $t$ be two points of $P$ such that $t$ lies in $C_{0}(s)$. If the top-right quadrant of $S(s)$ is empty and $s$ is t-protected, then there is an $s$-t-path in the $\theta_{4}$-graph of $P$ of length at most $18 \cdot d_{L_{1}}(s, t)$.

Proof. Since $s$ is $t$-protected, no point of $P$ lies above $s$, to the right of $s$ and below $\ell^{-}$; see the dark-shaded region in Fig. 5. Let $R$ be the smallest axis-aligned rectangle enclosing $s$ and $t$ and let $k$ be the number of $t$-protected points inside $R$, by the general position assumption, these points are strictly contained in $R$. We prove the lemma by induction on $k$.

Base case: Assume that $R$ contains no $t$ protected point, i.e., $k=0$. We claim that $R$ must be empty and we prove it by contradiction. Let $q$ be a point in $R$ and note that $q$ cannot lie above $\ell^{-}$as it would be $t$-protected yielding a contradiction. If $q$ lies below $\ell^{-}$, we can follow the $\max _{1}$-path from $q$ until reaching a $t$-protected point $p$ lying below $\ell^{-}$. Since $s$ is $t$-protected, $p$ must lie inside $R$ which is also a contradiction. Thus, $R$ must be empty.

Assume that $s_{0} \neq t$ since otherwise the result is trivial. As $R$ is empty and $s_{0} \neq t, s_{0}$ lies below $t$ and above $\ell^{+}$. Moreover, no point of $P$ lies above $t$, below

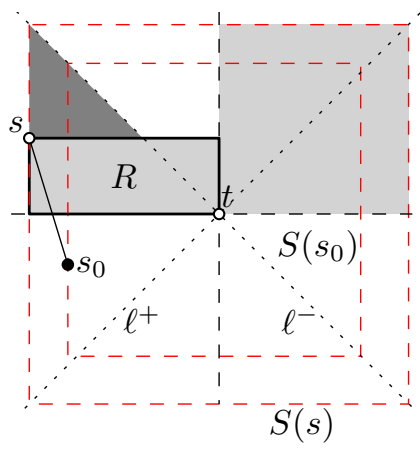

Figure 5: Base case. $\ell^{-}$and inside $S\left(s_{0}\right)$ since $s$ is $t$-protected. Thus, if we think of the set of points $P$ rotated 90 degrees clockwise around $t$, Lemma 8 and Lemma 9 guarantee the existence of an $s_{0}$ - $t$-path $\gamma$ of length at most $3 \cdot d_{L_{1}}\left(s_{0}, t\right)$. Since $s_{0}$ lies above $\ell^{+}, d_{L_{1}}\left(s, s_{0}\right) \leq d_{L_{1}}(s, t)$. Furthermore, $d_{L_{1}}\left(s_{0}, t\right) \leq 2 \cdot d_{L_{1}}(s, t)$ as $s_{0}$ lies inside $S(s)$. Thus, by joining $s s_{0}$ with $\gamma$, we obtain an $s$-t-path of length at most $7 \cdot d_{L_{1}}(s, t)$.

Inductive step: We aim to show the existence of a path $\gamma$ joining $s$ with a $t$-protected point $w \in R$ such that the length of $\gamma$ is at most $18 \cdot d_{L_{1}}(s, w)$. If this is true, we can merge $\gamma$ with the $w$ - $t$-path $\varphi$ existing by the induction hypothesis to obtain the desired $s$ - $t$-path with length at most $18 \cdot d_{L_{1}}(s, t)$. We analyze two cases depending on the position of $s_{0}$ with respect to $R$. 
Case 1. Assume that $s_{0}$ lies inside $R$. If $s_{0}$ lies above $\ell^{-}$, then $s_{0}$ is $t$-protected and hence we are done after applying our induction hypothesis on $s_{0}$. If $s_{0}$ lies below $\ell^{-}$, then we can follow its $\max _{1}$-path to reach a $t$-protected point $w$ that must lie inside $R$ as $s$ is $t$ protected. By running Algorithm 1 on $s$ and $w$, we obtain a path $\sigma_{s \rightarrow w}$ that goes through the edge $s s_{0}$ and then follows the $\max _{1}$-path of $s_{0}$ until reaching $w$; see Fig. 6 .

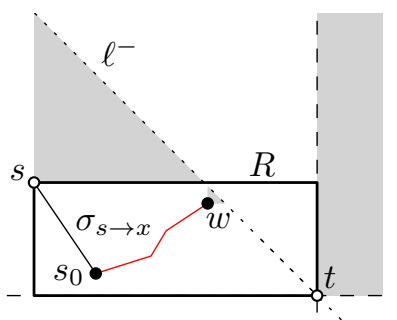

Figure 6: Case 1.

Since $s$ is $t$-protected and $w$ lies below $\ell^{-}, s$ is also $w$-protected. Therefore, Lemma 8 guarantees that $\left|\sigma_{s \rightarrow w}\right| \leq 3 \cdot d_{L_{1}}(s, w)$. By induction hypothesis on $w$, there is a $w$-t-path $\varphi$ such that $|\varphi| \leq 18 \cdot d_{L_{1}}(w, t)$. As $w$ lies in $R$, by joining $\sigma_{s \rightarrow w}$ with $\varphi$ we obtain the desired $s$-t-path of length at most $18 \cdot d_{L_{1}}(s, t)$.

Case 2. Assume that $s_{0}$ does not lie in $R$. This implies that $s_{0}$ lies below $t$. Assume also that $\sigma_{s \rightarrow t}$ does not reach $t$; otherwise we are done since $\left|\sigma_{s \rightarrow t}\right| \leq 3 \cdot d_{L_{1}}(s, t)$. Thus, as the top-right quadrant of $S(s)$ is empty, $\sigma_{s \rightarrow t}$ ends at a $t$-protected point $z$ lying in the bottom-right quadrant of $S(s)$. We consider two sub-cases depending on whether $\sigma_{s \rightarrow t}$ contains a point inside $R$ or not.

Case 2.1. If $\sigma_{s \rightarrow t}$ contains a point inside $R$, let $w$ be the first $t$-protected point of $\sigma_{s \rightarrow t}$ after $s$ and note that $w$ also lies inside $R$ since $s$ is $t$-protected. Notice that the part of $\sigma_{s \rightarrow t}$ going from $s$ to $w$ is in fact equal to $\sigma_{s \rightarrow w}$ since $w$ lies above $t$ and only 1-edges were followed after $s_{0}$ by Step 4 of Algorithm 1, see Fig. 7. Thus, as $s$ is also $w$-protected, the length of $\sigma_{s \rightarrow w}$ is bounded by $3 \cdot d_{L_{1}}(s, w)$ by Lemma 8 . Hence, we can apply the induction hypothesis on $w$ as

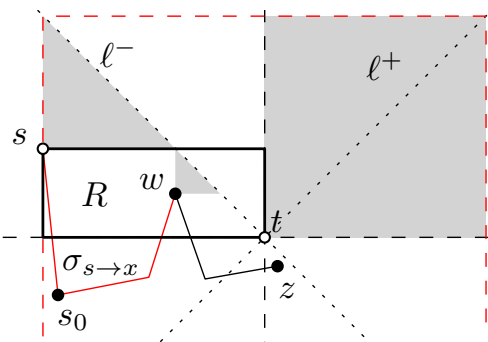

Figure 7: Case 2.1. before and obtain the desired $s$-t-path.

Case 2.2. If $\sigma_{s \rightarrow t}$ does not contain a point inside $R$, then $\sigma_{s \rightarrow t}$ follows only 1-edges from $s_{0}$ until reaching $z$ in the bottom-right quadrant of $S(s)$; see Fig. $8\left(\right.$ a). Let $P^{*}$ be the set of points obtained by reflecting $P$ on the line $\ell^{+}$. Since $z$ remains $t$-protected after the reflection, we can use Algorithm 1 to produce a path $\sigma_{z \rightarrow t}^{*}$ in the $\theta_{4}$-graph of $P^{*}$. Let $\gamma_{z \rightarrow t}$ be the path in the $\theta_{4}$ graph of $P$ obtain by reflecting $\sigma_{z \rightarrow t}^{*}$ on $\ell^{+}$. Note that $\gamma_{z \rightarrow t}$ ends at a point $w$ such that $w$ is either equal to $t$ or $w$ lies in the top-left quadrant of $S(s)$ since the top-right quadrant of $S(s)$ is empty. Since $z$ lies inside $S(s), d_{L_{1}}(z, t) \leq 2 \cdot d_{L_{1}}(s, t)$. Therefore, by Lemma 8 , the length of $\sigma_{s \rightarrow t} \cup \gamma_{z \rightarrow t}$ is given by

$$
\left|\sigma_{s \rightarrow t}\right|+\left|\gamma_{z \rightarrow t}\right| \leq 3 \cdot d_{L_{1}}(s, t)+3 \cdot d_{L_{1}}(z, t) \leq 3 \cdot d_{L_{1}}(s, t)+6 \cdot d_{L_{1}}(s, t)=9 \cdot d_{L_{1}}(s, t) .
$$

Two cases arise: If $\gamma_{z \rightarrow t}$ reaches $t(w=t)$, then we are done since $\sigma_{s \rightarrow t} \cup \gamma_{z \rightarrow t}$ joins $s$ with $t$ through $z$.

If $\gamma_{z \rightarrow t}$ does not reach $t(w \neq t)$, then $w$ lies below $\ell^{-}$by Lemma 9 applied on $\sigma_{z \rightarrow t}^{*}$. Moreover, as $s$ is $t$-protected, no point in $C_{1}(s)$ can be reached by $\gamma_{z \rightarrow t}$ and hence $w$ must lie inside $R$. We claim that $d_{L_{1}}(s, t) \leq 2 \cdot d_{L_{1}}(s, w)$. If this claim is true, $\left|\sigma_{s \rightarrow t} \cup \gamma_{z \rightarrow t}\right| \leq$ $9 \cdot d_{L_{1}}(s, t) \leq 18 \cdot d_{L_{1}}(s, w)$. Furthermore, by the induction hypothesis, there is a path $\varphi$ joining $w$ with $t$ of length at most $18 \cdot d_{L_{1}}(w, t)$. Consequently, by joining $\sigma_{s \rightarrow t}, \gamma_{z \rightarrow t}$ and $\varphi$, we obtain an $s$ - $t$-path of length at most $18 \cdot d_{L_{1}}(s, w)+18 \cdot d_{L_{1}}(w, t)=18 \cdot d_{L_{1}}(s, t)$. 

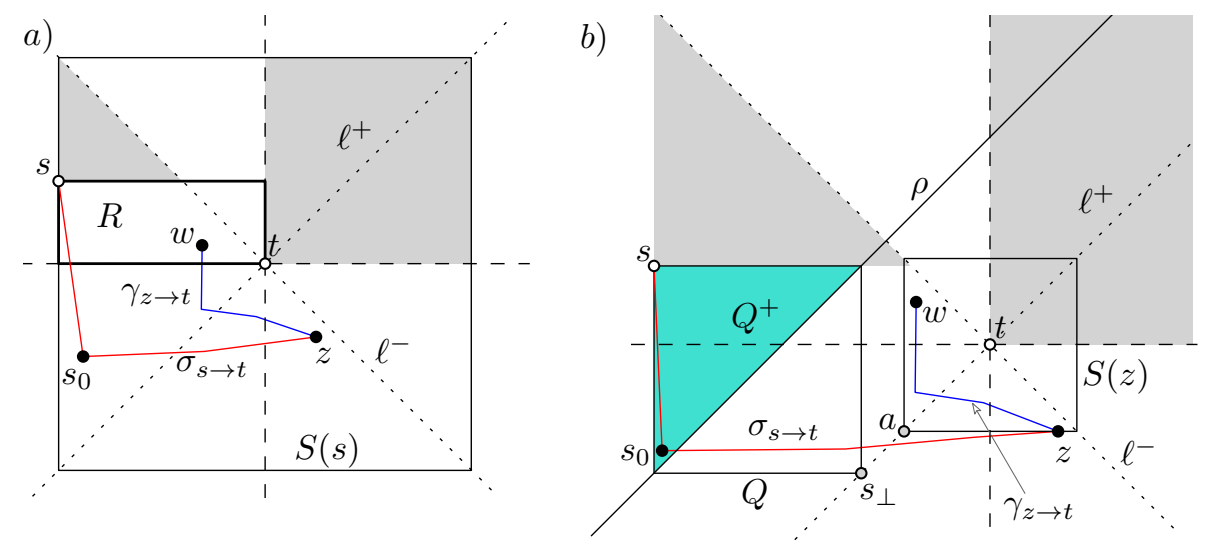

Figure 8: a) Case 2.2 in the proof of Lemma 1, path $\sigma_{s \rightarrow t}$ has no point inside $R$ and reaches a point $z$ lying in the bottom-right quadrant of $S(s)$. b) The inductive argument proving that the point $w$, reached after taking the path $\gamma_{z \rightarrow t}$, lies outside of the triangle $Q^{+}$containing all the points above $\rho$ and below $s$. As $s$ is $t$-protected, the region above $s$ and below $\rho$ is empty.

To prove that $d_{L_{1}}(s, t) \leq 2 \cdot d_{L_{1}}(s, w)$, let $s_{\perp}$ be the orthogonal projection of $s$ on $\ell^{+}$. Let $\rho$ be the perpendicular bisector of the segment $s s_{\perp}$ and notice that for every point $y$ in $C_{0}(s), d_{L_{1}}(s, t) \leq 2 \cdot d_{L_{1}}(s, y)$ if and only if $y$ lies below $\rho$.

Let $Q$ be the minimum axis-aligned square containing $s$ and $s_{\perp}$. Note that $\rho$ splits $Q$ into two equal triangles $Q^{+}$and $Q^{-}$as one diagonal of $Q$ is contained in $\rho$. Assume that $Q^{+}$is the triangle that lies above $\rho$. Notice that all points lying in $C_{0}(s)$ and above $\rho$ are contained in $Q^{+}$; see Fig. 8 (b). We prove that $w$ lies outside of $Q^{+}$and hence, that $w$ must lie below $\rho$.

If $s_{0}$ lies below $\rho$, then the empty triangle $\Delta_{0}(s)$ contains $Q^{+}$forcing $w$ to lie below $\rho$. Assume that $s_{0}$ lies above $\rho$. In this case, $z$ lies above $s_{0}$ as we only followed 1-edges to reach $z$ in the construction of $\sigma_{s \rightarrow t}$ by Step 4 of Algorithm 1. Let $a$ be the intersection of $\ell^{+}$and the ray shooting to the left from $z$. Notice that $w$ must lie to the right of $a$ as the path $\gamma_{z \rightarrow t}$ is contained in the square $S(z)$ and $a$ is one of its corners. As $z$ lies above $s_{0}$ and $s_{0}$ lies above $s_{\perp}$, we conclude that $a$ is above $s_{\perp}$ and both lie on $\ell^{+}$. Therefore, $a$ lies to the right of $s_{\perp}$, implying that $w$ lies to the right of $s_{\perp}$ and hence outside of $Q^{+}$. As we proved that $w$ lies below $\rho$, we conclude that $d_{L_{1}}(s, t) \leq 2 \cdot d_{L_{1}}(s, w)$.

\section{Lower Bound}

In this section we show how to construct a lower bound of 7 for the $\theta_{4}$-graph. We start with two vertices $u$ and $w$ such that $w$ lies in $C_{2}(u)$ and the difference of their $x$-coordinates is arbitrarily small. To construct the lower bound, we repeatedly replace a single edge of the shortest $u$-w-path by placing vertices in the corners of the empty triangle(s) associated with that edge. The final graph is shown in Fig. 9 .

We start out by removing the edge between $u$ and $w$ by placing two vertices, one inside $\Delta_{2}(u)$ and one inside $\Delta_{0}(w)$, both arbitrarily close to the corner that does not contain $u$ nor $w$. Let $v_{1}$ be the vertex placed in $\Delta_{2}(u)$. Placing $v_{1}$ and the other vertex in $\Delta_{0}(w)$ removed edge $u w$, but created two new shortest paths, $u v_{1} w$ being one of them. Hence, our next step is to extend this path.

We remove edge $v_{1} w$ (and its equivalent in the other path) by placing a vertex arbitrarily close to the corner of $\Delta_{1}\left(v_{1}\right)$ and $\Delta_{3}(w)$ that is farthest from $u$. Let $v_{2}$ be the vertex placed inside $\Delta_{1}\left(v_{1}\right)$. Hence, edge $v_{1} w$ is replaced by the path $v_{1} v_{2} w$. 
Next, we extend the path again by removing edge $v_{2} w$ (and its equivalent edge in the other paths). Like before, we place a vertex arbitrarily close to the corner of $\Delta_{0}\left(v_{2}\right)$ and $\Delta_{2}(w)$ that is farthest from $u$. Let $v_{3}$ be the vertex placed in $\Delta_{0}\left(v_{2}\right)$. Hence, edge $v_{2} w$ is replaced by $v_{2} v_{3} w$.

Finally, we replace edge $v_{3} w$ (and its equivalent edge in the other paths). For all paths for which this edge lies on the outer face, we place a vertex in the corner of the two empty triangles defining that edge. However, for edge $v_{3} w$ which does not lie on the outer face, we place a single vertex $v_{4}$ in the intersection of $\Delta_{3}\left(v_{3}\right)$ and $\Delta_{1}(w)$. In this way, edge $v_{3} w$ is replaced by $v_{3} v_{4} w$. When placing $v_{4}$, we need to ensure that no edge $u v_{4}$ is added as this would created a shortcut. This is easily achieved by placing $v_{4}$ such that it is closer to $v_{3}$ than to $w$. The resulting graph is shown in Fig. 9 .

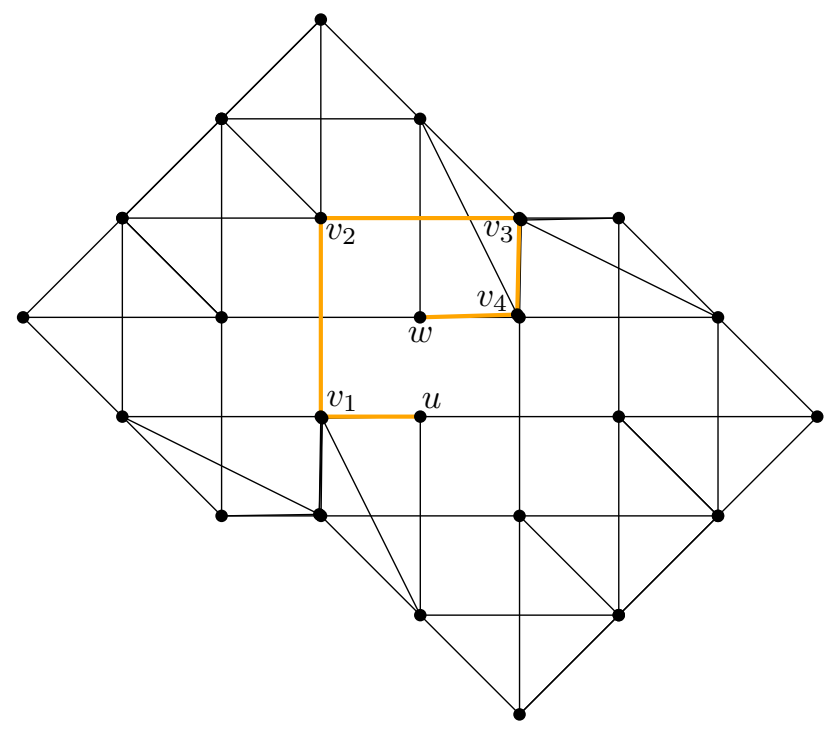

Figure 9: A lower bound for the $\theta_{4}$-graph. One of the shortest paths from $u$ to $w$ goes via $v_{1}, v_{2}, v_{3}$, and $v_{4}$.

Lemma 10. The stretch factor of the $\theta_{4}$-graph is at least 7.

Proof. We look at path $u v_{1} v_{2} v_{3} v_{4} w$ from Fig. 9. Edges $u v_{1}, v_{3} v_{4}$, and $v_{4} w$ have length $|u w|-\varepsilon$ and edges $v_{1} v_{2}$ and $v_{2} v_{3}$ have length $2 \cdot|u w|-\varepsilon$, where $\varepsilon$ is positive and arbitrarily close to 0 . Hence the stretch factor of this path is arbitrarily close to 7 .

\section{References}

[1] N. Bonichon, C. Gavoille, N. Hanusse, and D. Ilcinkas. Connections between theta-graphs, Delaunay triangulations, and orthogonal surfaces. In Proceedings of the 36th International Conference on Graph Theoretic Concepts in Computer Science (WG 2010), pages 266-278, 2010.

[2] N. Bonichon, C. Gavoille, N. Hanusse, and L. Perković. The stretch factor of $L_{1^{-}}$and $L_{\infty^{-}}$ Delaunay triangulations. In Proceedings of the European Symposia on Algorithms (ESA 2012), pages 205-216, 2012.

[3] P. Bose, M. Damian, K. Doüieb, J. O'Rourke, B. Seamone, M. Smid, and S. Wuhrer. $\pi / 2$-angle Yao graphs are spanners. International Journal of Computational Geometry \&3 Applications, 22(1):61-82, 2012. 
[4] P. Bose, P. Morin, A. van Renssen, and S. Verdonschot. The $\theta_{5}$-graph is a spanner. Computer Research Repository (CoRR), abs/1212.0570, 2012.

[5] K. Clarkson. Approximation algorithms for shortest path motion planning. In Proceedings of the 19th Annual ACM Symposium on Theory of Computing (STOC 1987), pages 56-65, 1987.

[6] N. M. El Molla. Yao spanners for wireless ad hoc networks. Master's thesis, Villanova University, 2009.

[7] J. Keil. Approximating the complete Euclidean graph. In Proceedings of the 1st Scandinavian Workshop on Algorithm Theory (SWAT 1988), pages 208-213, 1988.

[8] G. Narasimhan and M. Smid. Geometric Spanner Networks. Cambridge University Press, 2007.

[9] J. Ruppert and R. Seidel. Approximating the $d$-dimensional complete Euclidean graph. In Proceedings of the 3rd Canadian Conference on Computational Geometry (CCCG 1991), pages 207-210, 1991. 